\title{
Simulation et prédiction de la formabilité de la tôle via le simulateur emboutissage AutoForm
}

\author{
Simulation \& Prediction of sheet metal formability via AutoForm
}

stamping simulator

\author{
Oualid Kebdani ${ }^{1}$, Bouchaib Radi ${ }^{1}$ \\ ${ }^{1}$ Laboratoire Ingénierie, Management Industriel et Innovation, FST Settat, Université Hassan $1^{\mathrm{er}}$, Maroc.
}

\begin{abstract}
RÉSUMÉ. Au cours des dernières années, un énorme progrès a été réalisé dans l'utilisation industrielle des outils numériques pour la simulation des procédés de formage de la tôle. Cela est particulièrement vrai pour l'industrie automobile, afin de vérifier et de valider la faisabilité emboutissage. En effet pour atteindre les objectifs tout en respectant le deadline, le coût et la qualité dans le procédé de formage des tôles, une prédiction correcte de la formabilité au tout début du projet est devenue un facteur crucial. C'est la raison de la large application du CLF dans le but de mesurer et prédire les déformations limites, ainsi pour étudier l'influence de divers paramètres de matériau ou de procédé sur les chemins de déformations. Le présent article tente de donner une brève introduction au sujet du formage de la tôle par emboutissage et de décrire certains des défauts de formage qui peuvent se produire, ainsi le critère de rupture est présenté et appliqué à une pièce emboutie profonde via le simulateur Autoform.

ABSTRACT. In recent years, enormous progress has been made in the industrial use of digital tools with the aim to simulate the formability of sheet metal processes. This is especially true for the automotive industry, in order to verify and validate stamping feasibility. Indeed cost and quality targets in industrial sheet metal forming processes, correct formability prediction in a very early project stage has become a crucial factor. This is the reason for the wide application of FLC for the purpose of measuring and predicting limit strains, thus to study the influence of various materials or process parameters on strain paths. This article attempts to give a brief introduction to the subject of stamping sheet metal forming and describe some of the forming defects that can occur, thus the failure criterion is presented and applied to a deep drawn part via the Autoform simulator.

MOTS-CLÉS. Emboutissage profond, simulation numérique, méthode des éléments fini, comportement mécanique de la tôle, diagramme Limite de Formage.

KEYWORDS. Deep drawing, numerical simulation, Finite element method, Mechanical behavior of sheet-metal, Forming limit diagram.
\end{abstract}

\section{Introduction}

Le principal avantage du processus d'emboutissage est sa productivité élevée, qui est une qualité très importante dans l'industrie automobile hautement efficace et automatisée. Les pièces en tôle de divers niveaux de complexité sont produites rapidement, souvent en très gros volume, à l'aide d'un outillage d'emboutissage.

Cependant, les procédés d'emboutissage et la conception des outillages associés reposent sur des connaissances techniques issues de l'expérience et des standards métiers mis en œuvre depuis plusieurs décennies, d'où une démarche expérimentale par essai-erreur-correction est nécessaire afin de valider le produit fini. Par ailleurs, les délais de conception sont plus longs et les modifications en cours d'industrialisation sont très coûteuses.

Le développement des codes de calcul et la simulation numérique a pour objectif de décrire et préciser les spécificités de conception entre les différents intervenants d'un projet dans la mesure d'offrir la possibilité de vérifier la faisabilité des formes envisagées dès la phase d'avant-projet afin de faciliter la réalisation et les modifications des pièces par rapport à des paramètres d'entrées données et simulés dans la mesure de concevoir et industrialiser bien de la première tentative pour réduire les investissements [AND 04]. Ce travail permet de prédire le risque de striction/rupture, de déterminer les zones à fort amincissement local avant une déchirure et de comprendre l'influence des différents paramètres de ce procédé sur le comportement des matériaux. 


\section{Procédé de l'emboutissage profond}

\subsection{Définition}

L'emboutissage est un procédé d'obtention de pièce à partir de tôle découpée et déformée mécaniquement au moyen d'outils. Cette déformation s'effectue «à froid »: les outils et la tôle sont à température ambiante [RAD 16]. Il existe des variantes dites « à chaud » entre $300^{\circ}$ et $900^{\circ}$ intermédiaires entre l'emboutissage à froid et le forgeage. Ce procédé permet d'obtenir des pièces de formes complexes le plus souvent non-développables, contrairement aux procédés tels que le pliage ou le roulage. Les pièces embouties se trouvent majoritairement parmi les pièces de la structure caisse (Pavillon, côté d'habitacle, plancher), les ouvrants (panneau de porte, capot, coffre), les pièces de liaisons au sol (train AV, AR).

\subsection{Schéma du principe de l'emboutissage}

La pièce est formée à l'aide d'estampes (poinçons, matrices et serre-flan), entre lesquelles est déformée une feuille de métal.

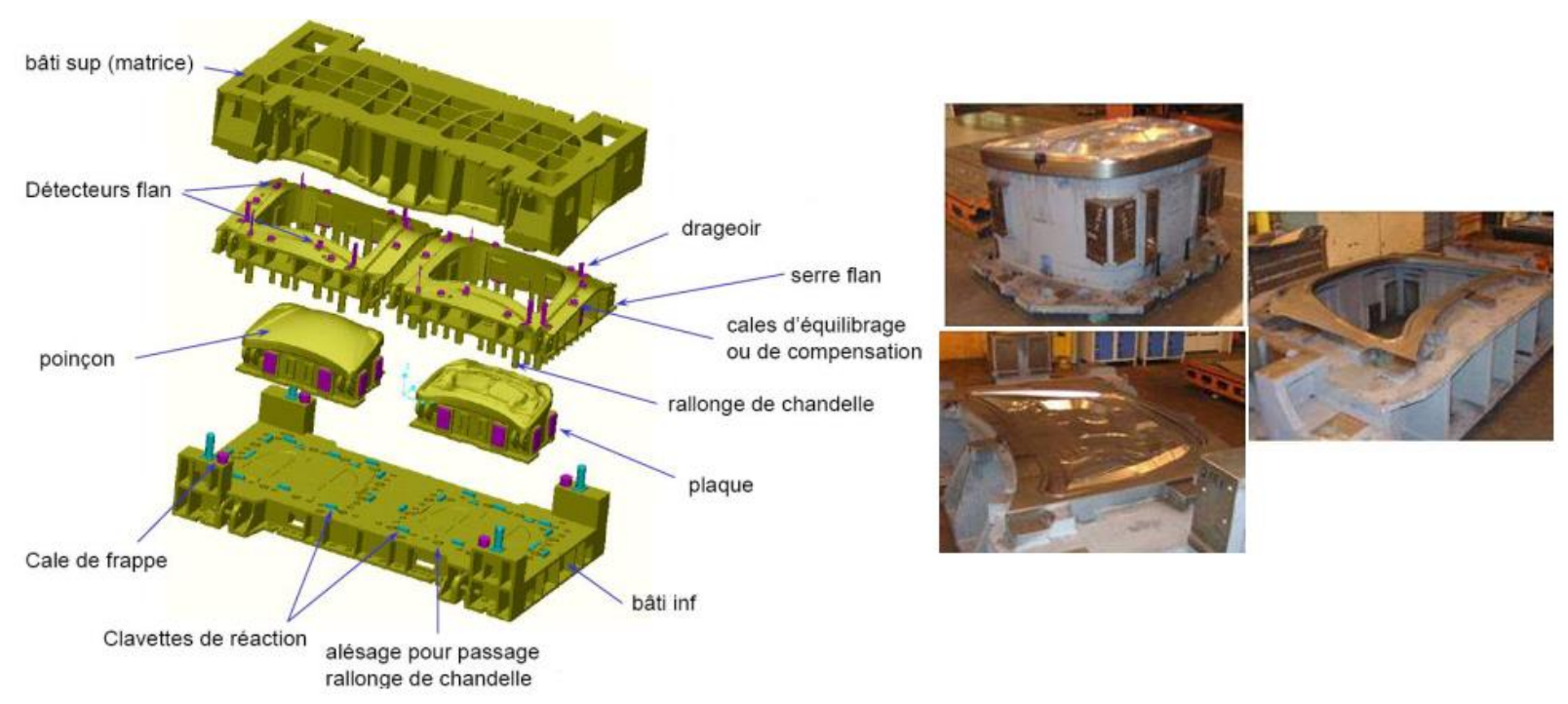

Figure 1. Outil d'emboutissage

Le principe générique simplifié du procédé d'emboutissage peut être représenté comme suit :
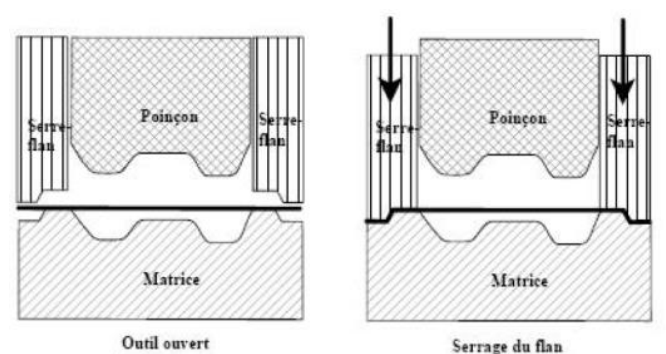

Serrage du flan

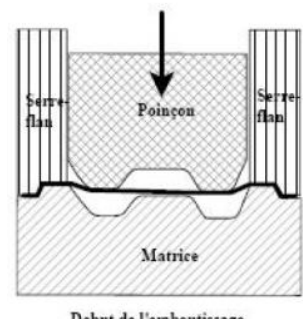

Debut de l'emboutissage
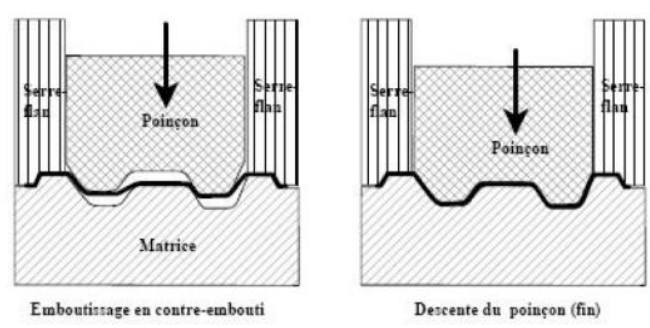

Figure 2. Principe du procédé d'emboutissage 


\section{3. Élément nécessaire dans l'emboutissage profond}

\subsection{Description des surfaces de l'outil}

La figure 3 décrit les différentes zones de l'outil d'emboutissage qui le caractérise :

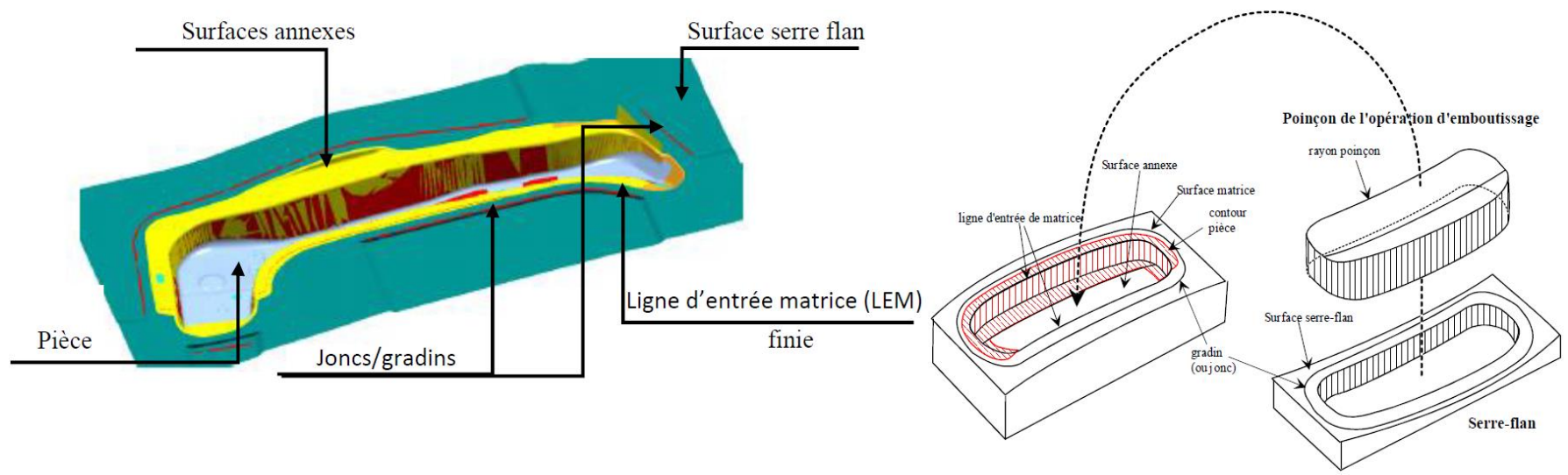

Figure 3. Méthodologie pour construire la surface annexe

La LEM est le point de départ de la construction des surfaces annexes, c'est le contour de la pièce avec son habillage qui sépare les surfaces annexes de la surface serre flan, elle peut être modifiée au fur et à mesure de la détermination des surfaces annexes et de l'évolution de la gamme.

\subsection{Contraintes dans le procédé d'emboutissage profond}

L'emboutissage impose, à la tôle, divers modes de déformation. Les deux cas limites rencontrés, dans la pratique, étant : L'expansion et le rétreint. Pratiquement, l'emboutissage par expansion a lieu lorsque le flan est bloqué entre le serre-flan et la matrice, toute la déformation résulte d'une tractiontraction et intervient sous le poinçon quelle que soit la forme. Elle se caractérise par une rupture ou casse en cas d'allongement excessif et striction l'amincissement localisé juste avant la rupture.

L'emboutissage par rétreint ou « emboutissage profond » a lieu lorsque le flan est libre de glisser entre la matrice et le serre-flan. La déformation commence sous le serre-flan en compression-traction et se poursuit dans l'entrée de la matrice en traction. Elle se caractérise de son tour par de défauts d'aspect visible à l'œil nu comme le pli ou une ondulation. Tout l'art de l'emboutissage est de réaliser le meilleur compromis entre les déformations des deux types en jouant sur les divers paramètres qui contrôlent le déplacement du métal dans l'outil [HAM 16, DRO 18].

\subsection{Fonctions du Serre-Flan}

Le serre-flan a pour rôle de presser le flan contre la matrice et de contrôler le flux de matériau dans la cavité de la matrice pendant la course grâce à des forces de friction et d'empêcher l'apparition anarchique de plis. Ce sont des aménagements de retenue de matière, le freinage de la matière limite alors l'alimentation des emboutis en matière, ce freinage permet d'avoir un meilleur tendu et un gain en plasticité, on distingue : les gradins (ou redan) et les joncs.

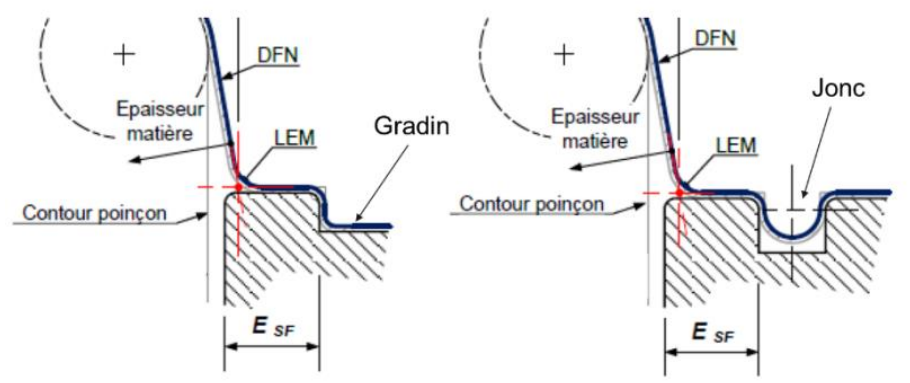

Figure 4. Moyen de freinage Jonc, Gradin et baguette 


\section{Comportement mécanique de la mise en forme des matériaux}

Il existe différents modèles pour modéliser le comportement des matériaux. Les modèles de VonMises et Tresca supposent que le matériau est homogène, c'est-à-dire que les propriétés du matériau sont supposées identiques en tout point de la pièce. En réalité, la microstructure de la tôle dépend au sens de l'opération de laminage, ce qui entraîne la dépendance directionnelle des propriétés plastiques (valeur r). La figure 9.a montre le critère d'élasticité Hill, Barlat et Banabic pour définir correctement la surface d'élasticité anisotrope. Les critères les plus importants pour une modélisation adéquate des matériaux sont le coefficient d'écrouissage, d'anisotropie, la limite d'élasticité et la limite de rupture du matériau [ABB 85, BEN 17].

\subsection{Anisotropie}

Couramment, six valeurs d'entrée sont utilisées pour l'identification des paramètres du matériau, représentées dans la figure 9.b : les trois contraintes d'élasticité $(\sigma 0, \sigma 45, \sigma 90)$ et les trois valeurs d'anisotropie r. L'anisotropie est la variation de la microstructure entre la largeur et l'épaisseur, dans le sens de laminage, obtenues à partir d'essais de traction uni-axiaux (réalisés avec des orientations de $0^{\circ}$, $45^{\circ}$ et $90^{\circ}$ par rapport au sens de laminage), calculé à l'aide de la formule :

$r=\frac{\varepsilon_{w}}{\varepsilon_{t}}=\frac{\ln \left(\frac{w}{w_{0}}\right)}{\ln \left(\frac{t}{t_{0}}\right)}$

$\varepsilon_{\mathrm{t}}$ Déformation dans l'épaisseur

$\varepsilon_{\mathrm{w}}$ Déformation dans la largeur

\section{2. Écrouissage}

Afin de définir le comportement d'écrouissage de la tôle, nous avons décidé d'utiliser la loi d'écrouissage de Ludwik. Dans le modèle de la loi de Ludwik, trois constantes de matériau doivent être estimées par la courbe rationnelle contrainte-déformation (voir figure 9.c) : le coefficient de résistance $(\mathrm{K})$, l'exposant d'écrouissage $(\mathrm{n})$ et la contrainte initiale $\left(\sigma_{0}\right)$ avant de se limiter à l'équation suivante :

$$
\text { Ludwik }=\sigma_{0}+K \varepsilon^{n}\left(\sigma_{0} \text { limite élastique }\right)
$$

\subsection{Essai de traction uni-axial}

Les propriétés mécaniques du matériau ont été mesurées sur l'essai de traction dans trois directions $\left(0^{\circ}, 45^{\circ}\right.$ et $\left.90^{\circ}\right)$ liées au sens de laminage. Les propriétés mécaniques évaluées à partir de l'essai de traction uni-axiale sont la déformation d'ingénierie (e) et la ou les contraintes d'ingénierie, ces propriétés sont obtenues directement à partir des mesures suivantes :

$$
e=\frac{\left(L_{1}-L_{0}\right)}{L_{0}}=\frac{\Delta L}{L_{0}} \quad \text { et } \quad \varepsilon=\ln \left(\frac{L_{1}}{L_{0}}\right)
$$

- $\mathrm{L}_{0}$ : longueur initiale

- $\mathrm{L}_{1}$ : longueur après déformation

- e : déformation ingénieure

- $\varepsilon$ : déformation vraie

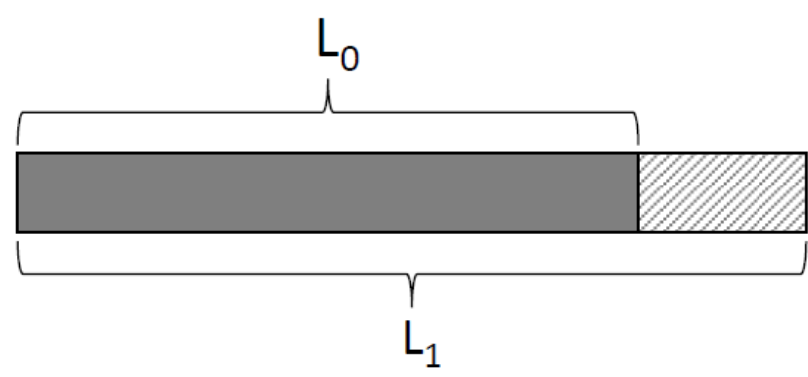

Les déformations principales sont les déformations dans les directions principales (i.e. traction/compression). Elles sont représentées par les variables de résultat Major Strain et Minor Strain dans AutoForm et sont toujours perpendiculaires les unes aux autres [RAD 07]. 


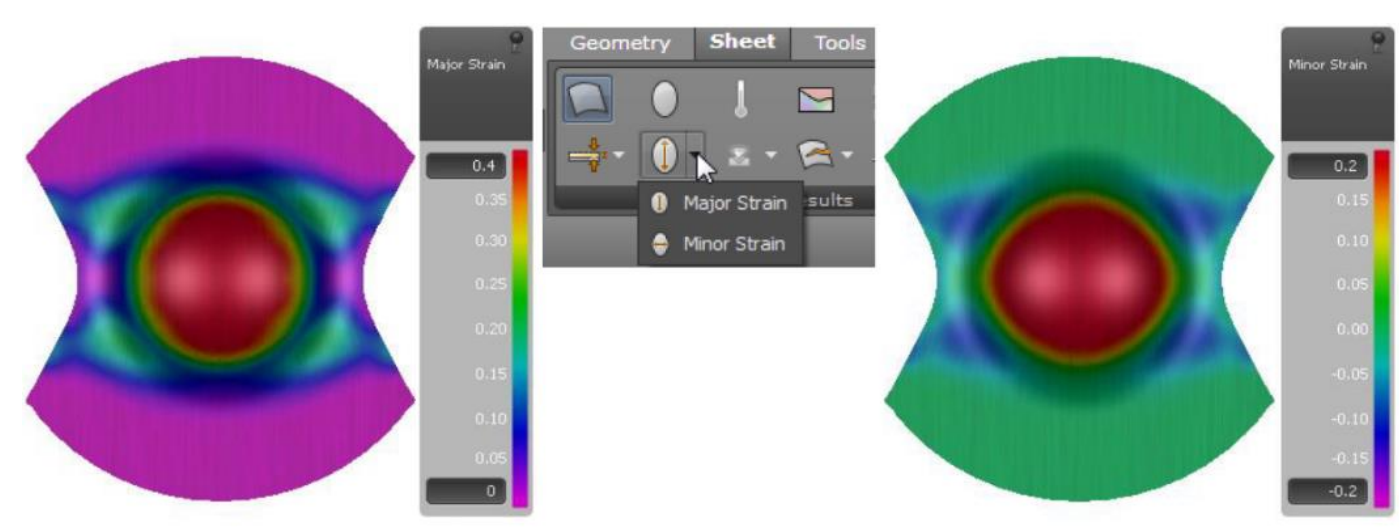

Principle Strain Direction

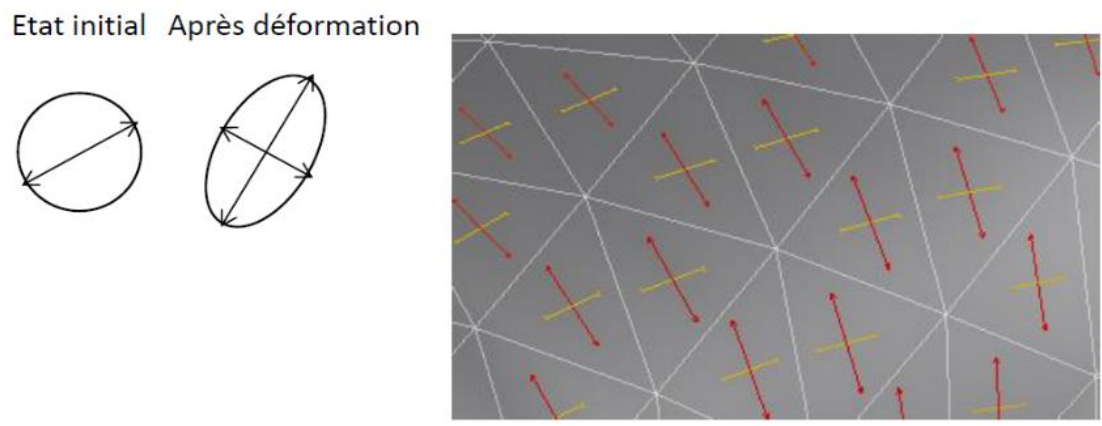

Figure 5. Les déformations principales

Finalement, le comportement de contrainte d'écoulement a été obtenu en calculant la déformation vraie $(\varepsilon)$ et la contrainte vraie $(\sigma)$. La courbe de contrainte des déformations est illustrée sur la figure 9.d.

$\varepsilon=\ln (1+e) \quad$ et $\quad \sigma=s(1+e)$

\subsection{Courbes limite de formage}

Des éprouvettes de différentes formes produisent des états (chemins) de déformation différents. Si l'éprouvette est sollicitée jusqu'à apparition de la striction, un état de déformation critique peut être déterminé pour chacun de ces chemins.

Les courbes limites de formage (CLF) définissent la capacité de déformation par emboutissage d'un matériau selon différents modes de sollicitation (de la déformation «pleine tôle » à l'emboutissage avec avalement). Pour chacun de ces modes, la limite est marquée par l'apparition d'un amincissement localisé (striction) ou d'une rupture [OZT 04].
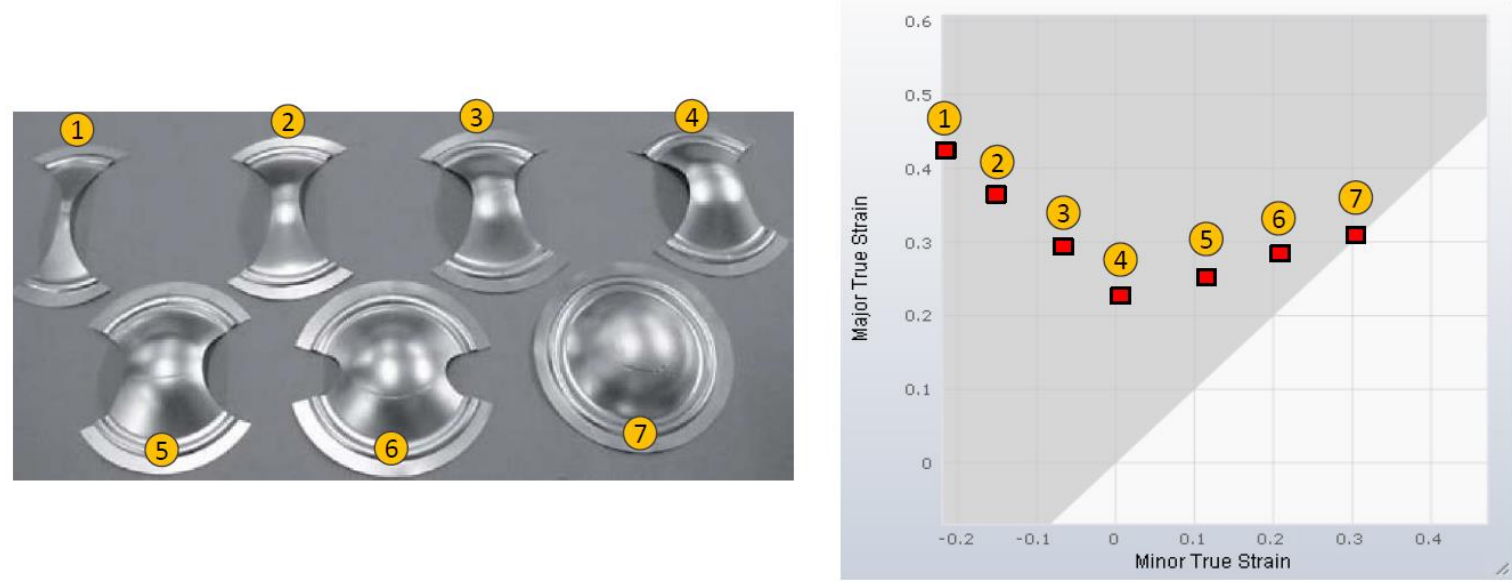

Figure 6. Points de défaillance du CLF 
La connexion des points de défaillance donne la courbe limite de formage (c'est-à-dire la limite de formage potentielle). La courbe limite de formage est un paramètre de matériaux qui doit être déterminé individuellement.

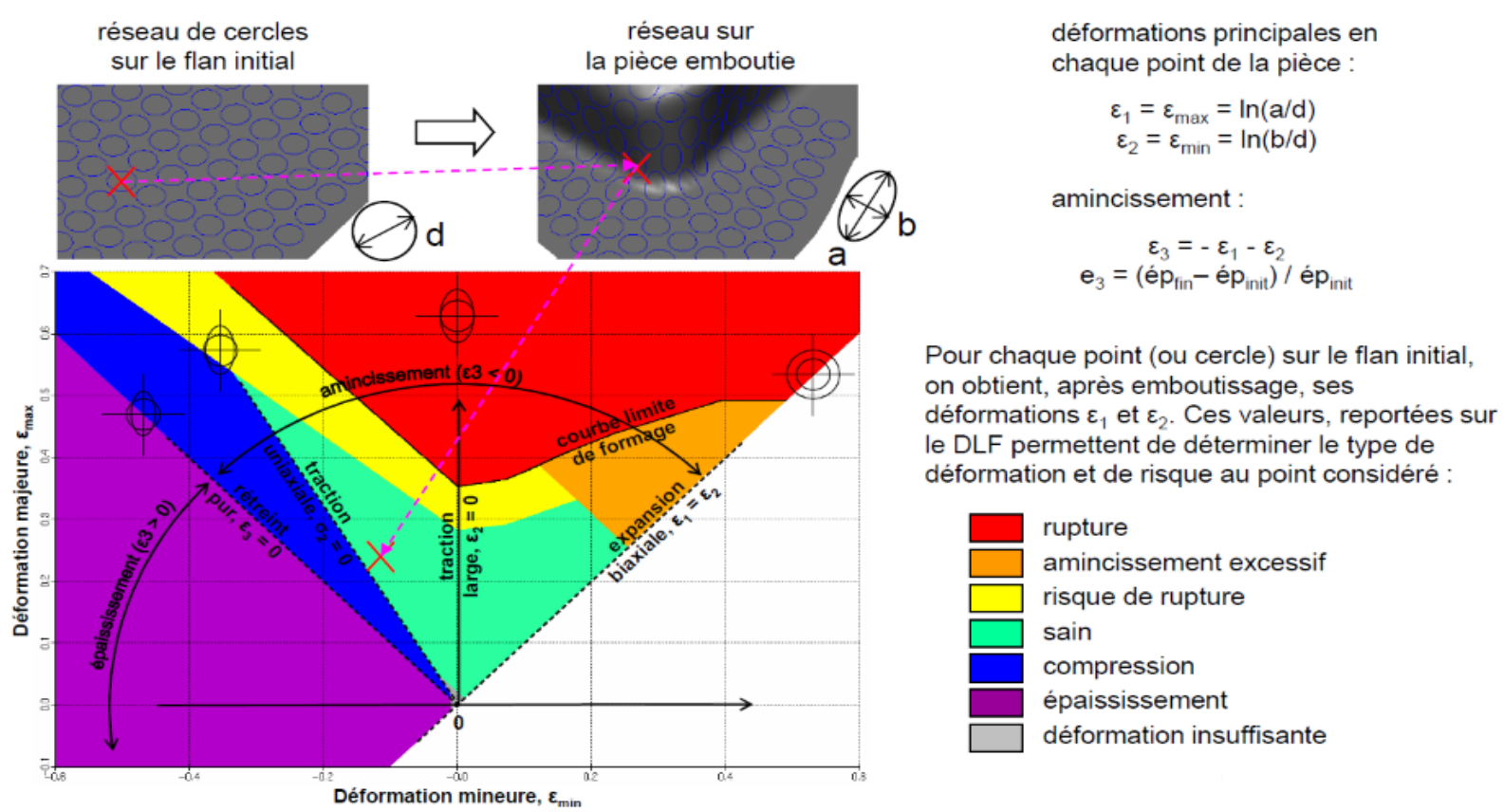

Figure 7. Diagramme et Courbe Limite de Formage (DLF et CLF)

Une évaluation qualitative de la pièce formée peut être réalisée par l'état de déformation. Cette évaluation peut être représentée par différentes couleurs dans la courbe limite de formage.

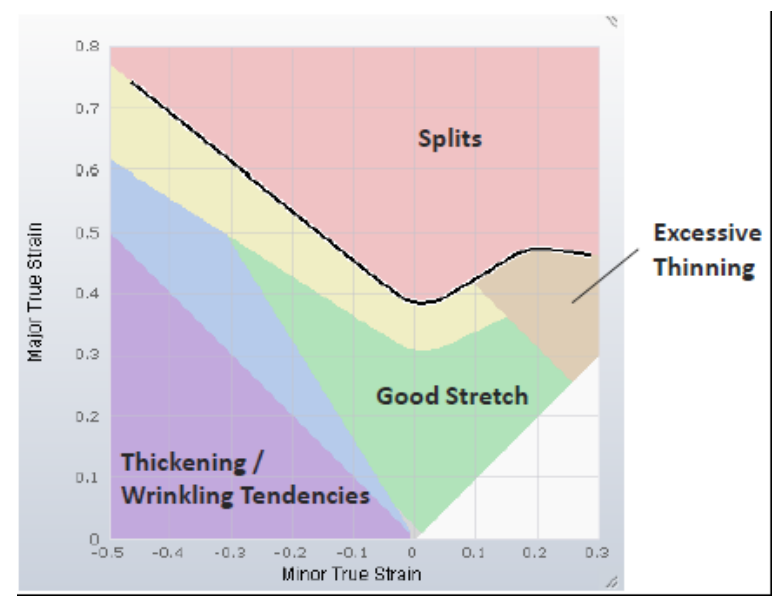

Figure 8. Code couleurs représente le Diagramme Limite de Formage

La zone située au-dessus des points de défaillance de la courbe est colorée en rouge, des criques sont attendues dans cette zone. En raison des variations des propriétés du matériau et de la précision de mesure lors de la détermination de la courbe, une marge de sécurité de $20 \%$ en dessous de la courbe est appliquée, cette marge de sécurité est colorée en jaune.

\section{Formulations des éléments finis via AUTOFORM}

Au fil des ans, un certain nombre de formulations des EF ont été présentées dont l'objectif est la simulation de la mise en forme de la tôle. La relation constitutive la plus couramment utilisée dans les contextes de formage de tôles est le modèle de Hill1 de 1948. Il peut décrire une anisotropie orthonormée du matériau. 
L'objectif est que le code de simulation soit capable de simuler le processus de formage complet et de pouvoir révéler d'éventuels défauts. Il devrait également être dans la mesure de faire varier tous les paramètres d'entrés, qui sont utilisés dans la réalité pour optimiser le processus d'emboutissage. Cela évite la très coûteuse procédure empirique dite essai-erreur-correction et permet ainsi de réaliser des économies substantielles et de réduire significativement les temps de conception de nouvelles gammes [MAT 00, MAK 98].

Le logiciel AutoForm a été développé pour modéliser les processus d'emboutissage profond, il s'appuie sur l'approche des éléments finis : (la méthode statique implicite) [KUB 95]. Dans le programme, les descriptions mathématiques des propriétés des matériaux sont adaptées aux simulations d'emboutissage profond. Les données et les graphiques énumérés ci-dessus (figures 1,) ont été utilisés comme données d'entrées dans la simulation numérique du comportement de la feuille métallique. Le point d'entrée au simulateur Autoform commence par injecter le fichier CAO qui représente la géométrie souhaitée comme le montre la figure 11, les paramètres du matériau et du process conçu.

\section{Résultat de simulation et discussion}

\subsection{Données entrées de la simulation}

\subsubsection{Matière première utilisée}

Afin de d'illustrer et analyser les résultats possibles via Autoform, une simulation d'une pièce automoblile Gousset gouttière aile ar a été étudiée. Le matériau utilisé pour cette composante automobile est un acier doux avec une bonne formabilité [LOU 12]. Les données du matériau se trouvent dans le tableau 1.

\begin{tabular}{|c|c|c|c|c|c|c|c|c|c|c|c|c|c|c|}
\hline \multirow{2}{*}{$\begin{array}{l}\text { Thickness } \\
(\mathbf{m m})\end{array}$} & \multicolumn{2}{|c|}{ Rpo,2 } & \multicolumn{2}{|c|}{$\mathbf{R m}$} & \multirow{2}{*}{$\begin{array}{l}\text { A\% } \\
\text { mini }\end{array}$} & \multirow{2}{*}{\begin{tabular}{|c|}
$\mathrm{n}$ \\
mini \\
\end{tabular}} & \multirow{2}{*}{$\begin{array}{c}\mathbf{r} \\
\text { mini }\end{array}$} & \multirow{2}{*}{$\begin{array}{c}\text { Coefficient } \\
\text { de poisson } \\
\mathbf{v}\end{array}$} & \multicolumn{3}{|c|}{$\begin{array}{l}\text { Yield strength } \\
\text { [Mpa] }\end{array}$} & \multicolumn{3}{|c|}{ r-value } \\
\hline & mini & Iaxi & ini & $\operatorname{maxi}$ & & & & & $0^{\circ}$ & $45^{\circ}$ & $90^{\circ}$ & $0^{\circ}$ & $45^{\circ}$ & $90^{\circ}$ \\
\hline$<1,47$ & 160 & 200 & 280 & 340 & 37 & 0,19 & 1,80 & 0,3 & 176 & 193 & 178,6 & 1,87 & 1,49 & 2,04 \\
\hline
\end{tabular}

Table 1. Les propriétés du matériau de la nuance ES

Le Flanc utilisé est une tôle en acier ES matériau élasto-plastique anisotrope (en appliquant l'algorithme de plasticité anisotrope de Hill), Module de Young : $\mathrm{E}=210 \mathrm{GPa}$, épaisseur $=1,05 \mathrm{~mm}$, Limite Élastique : $\mathrm{Re}=210 \mathrm{MPa}$, Limite de rupture Rm: 307,7 MPa, Allongement : A = 24,4\%. La figure ci-contre montre la modélisation du matériau choisi sous AutoForm [FED 17] : 


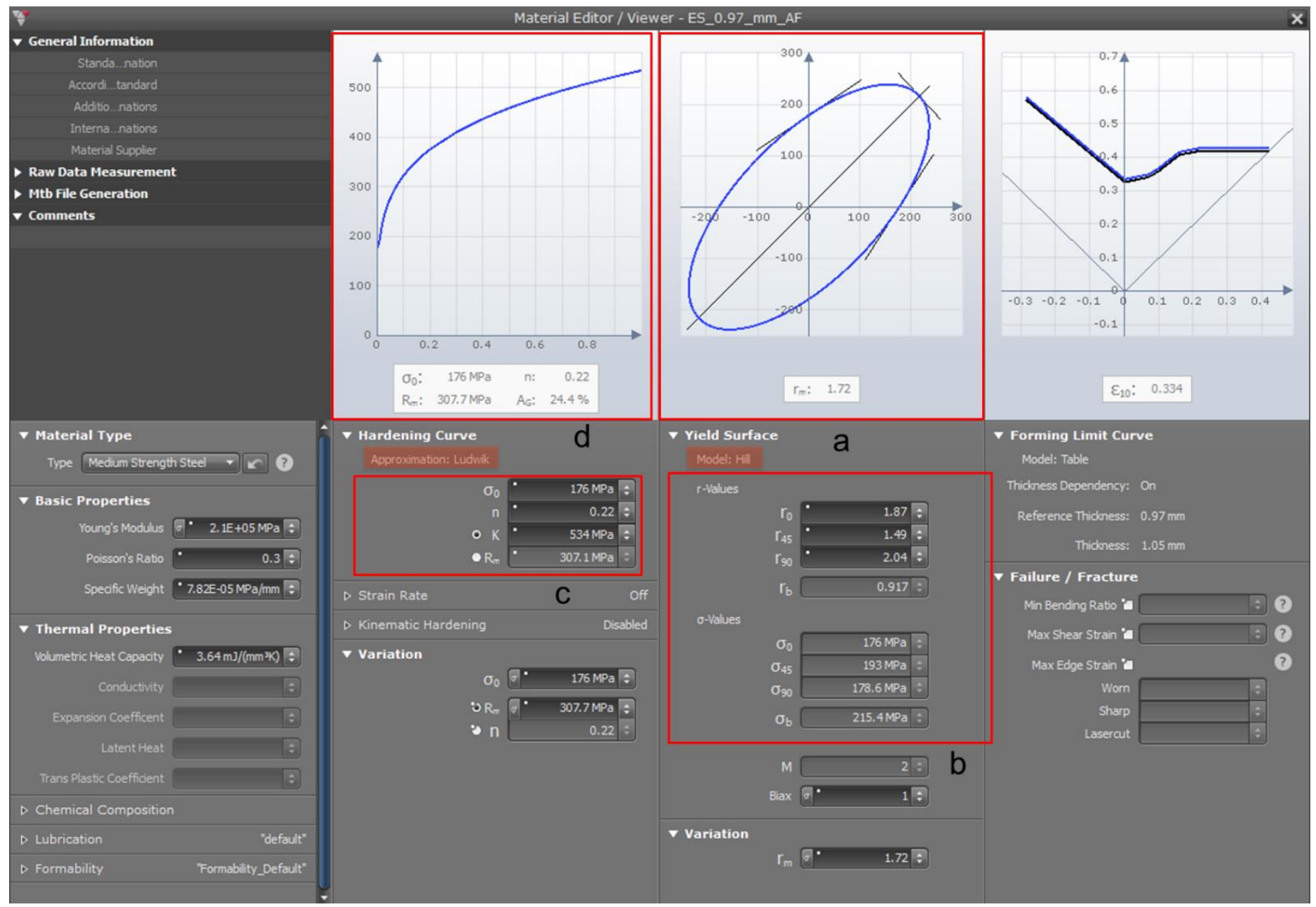

Figure 9. Modélisation de matériau sous AutoForm

Le Flan est cisaillé trapèze, il a comme $\mathrm{LB}=1140 \mathrm{~mm}$ et pas de découpe $=590 \mathrm{~mm}$.

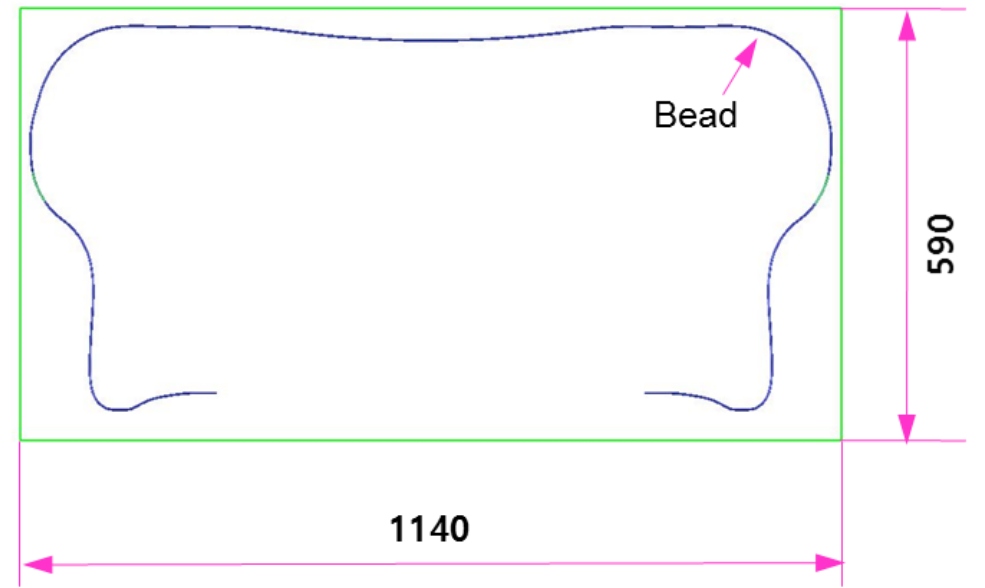

Figure 10. Dimension du Flan

\subsubsection{Process choisi}

La simulation en emboutissage de la tôle nécessite des paramètres process appropriés, tels que les différents composants d'un outil (poinçon, matrice et serre-flan), les joncs de retenu, les forces, etc. Une vue isométrique de la pièce embouti via des différents cotés. 

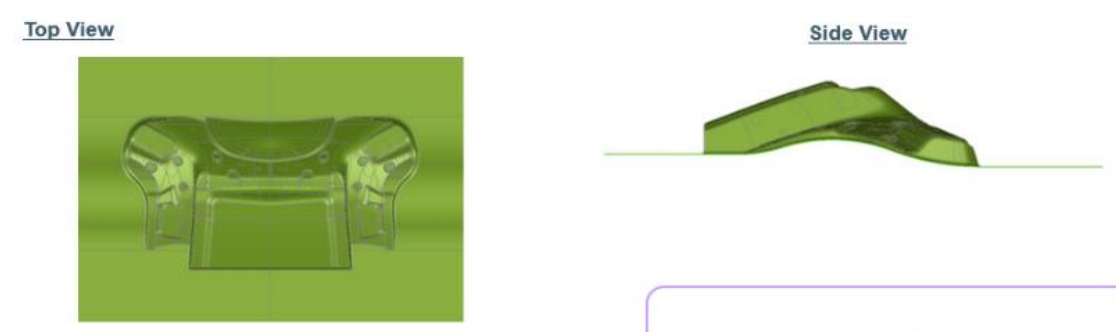

Front View
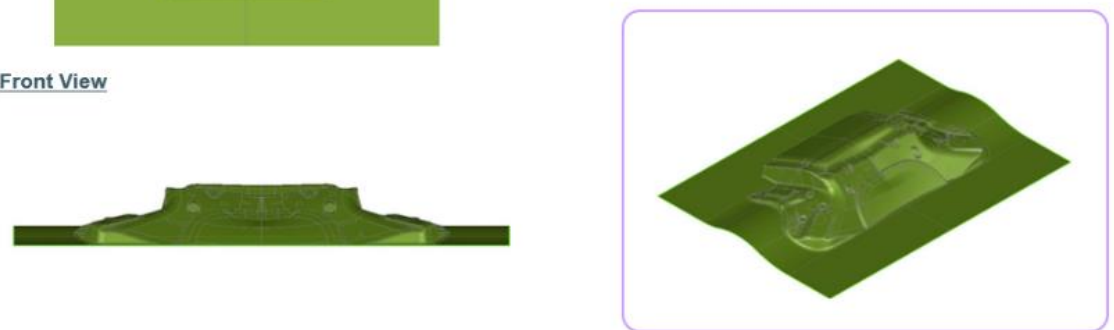

Figure 11. Vue isométrique de la pièce

Les joncs de retenu sont présentés autour de la pièce dans les surfaces annexes comme suit :

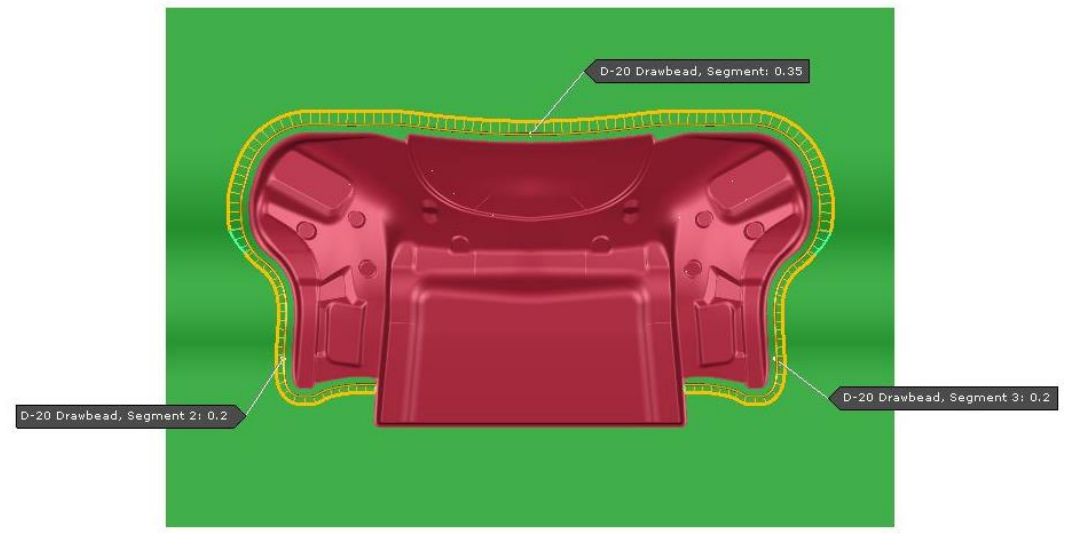

Figure 12. Dimension du Jonc de retenu

\subsubsection{Détermination des efforts appliqués}

Afin de dimensionner la gamme d'emboutissage de manière précise, pour dimensionner les lignes sur lesquelles sera montée la gamme, il est indispensable de déterminer quelles forces sont nécessaires pour former la pièce. Les données de ces efforts peuvent être obtenues à partir des résultats de la simulation.

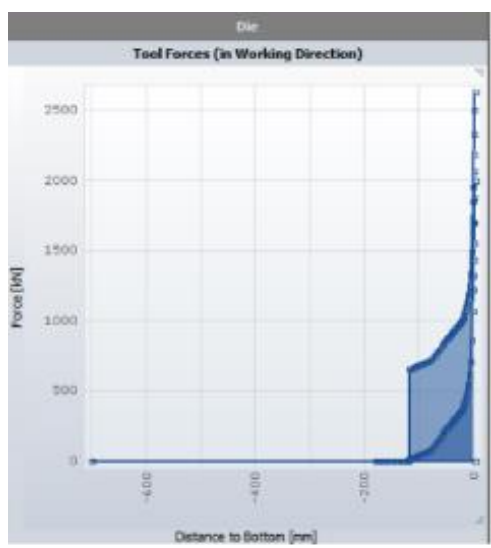

DIE : 366.8 TON

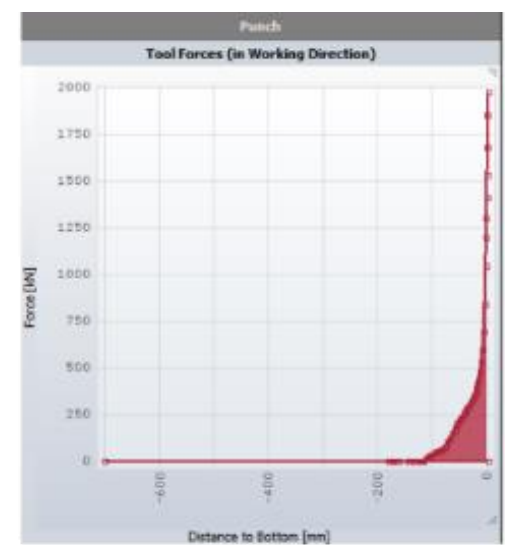

PUNCH : 275.8 TON

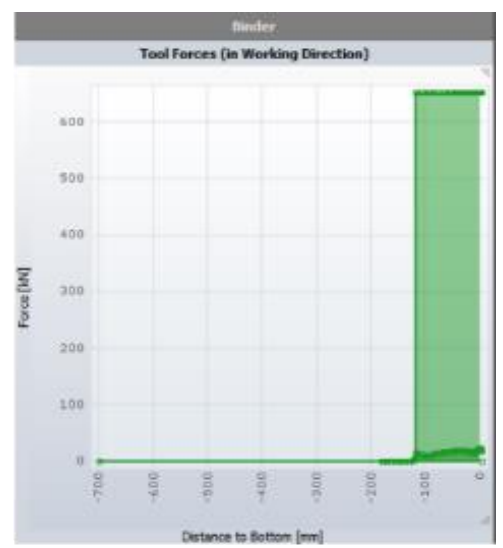

$\mathrm{BH}: 65 \mathrm{TON}$

Figure 13. Efforts serre-flan, poinçon et matrice

La force de serrage du flan choisi pendant la simulation numérique ainsi que pendant la réalisation de la pièce est $650 \mathrm{kN}$. 


\subsection{Résultats attendus et discussion}

À l'aide d'AUTOFORM, la gamme d'emboutissage est conçu, les paramètres tels que la géométrie du flan, le matériau, les forces, ainsi que l'emplacement et la géométrie des joncs de serrage sont adaptés. Par conséquent, la simulation a été réalisée afin de déterminer l'influence différents paramètres sur la formabilité résultante. Le flan est totalement embouti lors de la première opération d'emboutissage.

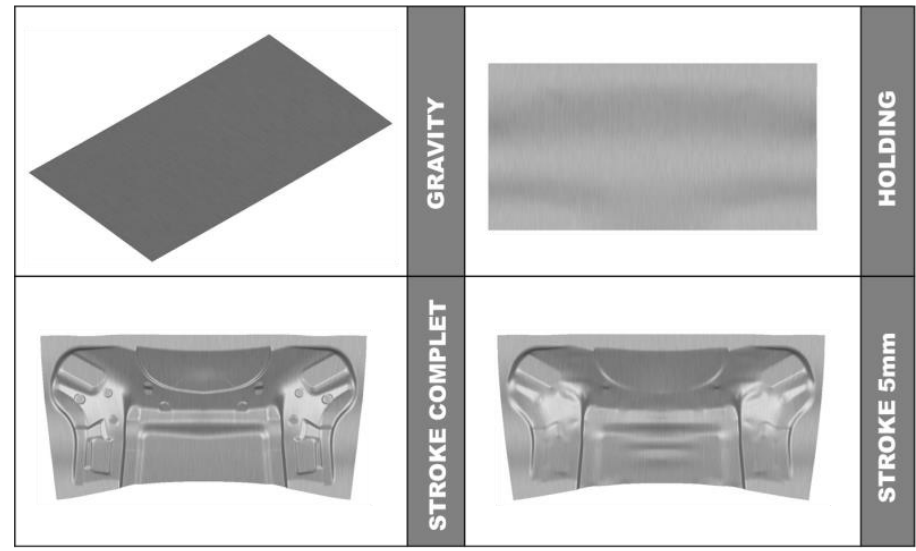

Figure 14. Opération d'emboutissage complet

\subsubsection{Formabilité}

Le risque de rupture est apprécié grâce à l'utilisation de la Courbe Limite de Formage (CLF) et du Diagramme Limite de Formage (DLF). La figure ci-contre présente les résultats obtenus lors de la simulation au nominal avec une force de serrage de $65 \mathrm{~T}$ :

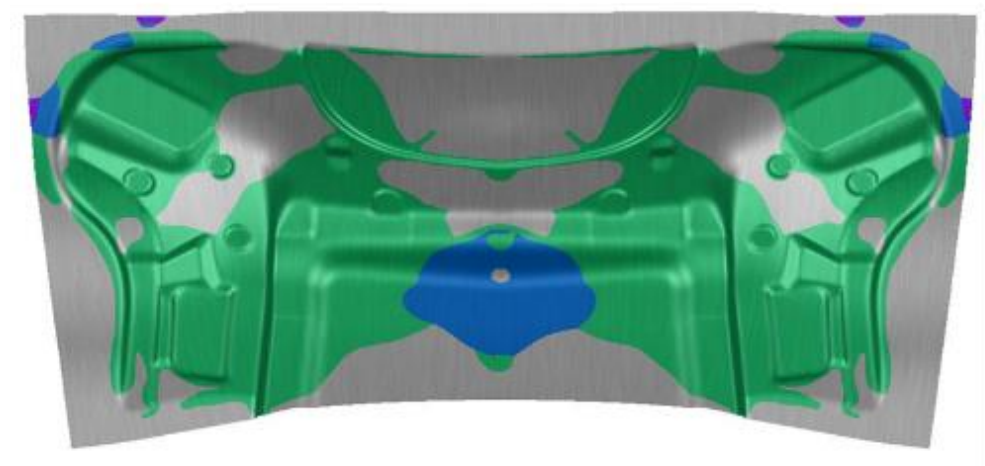

Forming Limit Diagram (Linear)
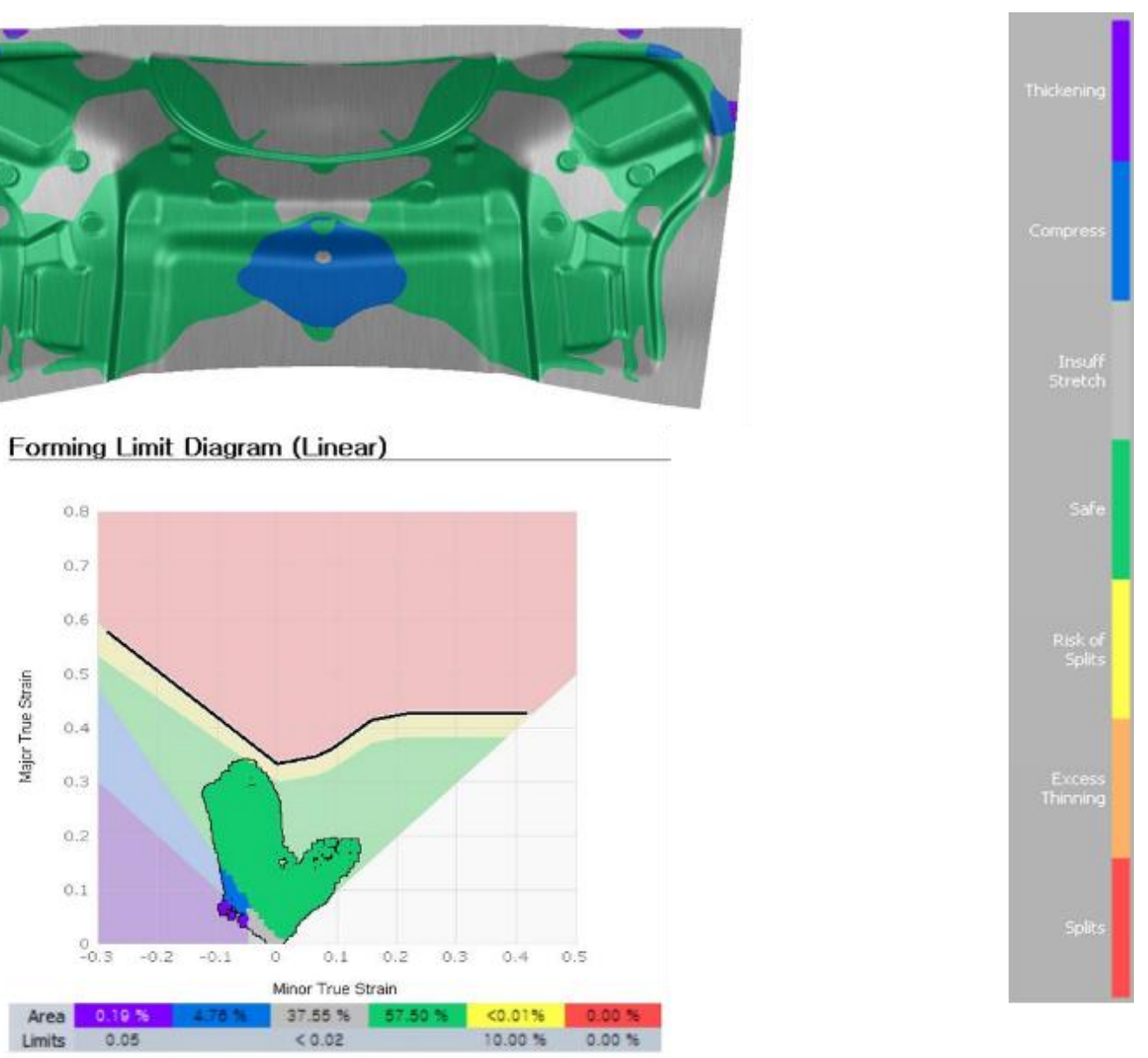

Figure 15. Diagramme Limite de Formage au nominal 
La figure 15 et 16 montrent les différentes zones du FLD : 58\% des éléments sont dans la zone saine, $5 \%$ des éléments sont sous la compression et $<0,01 \%$ des éléments sont sous l'étirement insuffisant. Ces résultats montrent que le processus d'emboutissage profond conçu reste non-robuste, vu qu'il y a un léger amincissement de $0,01 \%$ qui peut générer un risque de rupture dans la zone 1 à $2 \mathrm{~mm}$ du PMB (Point mort bas) et une casse dans la zone 2 a $10 \mathrm{~mm}$ du PMB. Ainsi un peu de compression au tour des joncs de freinage.

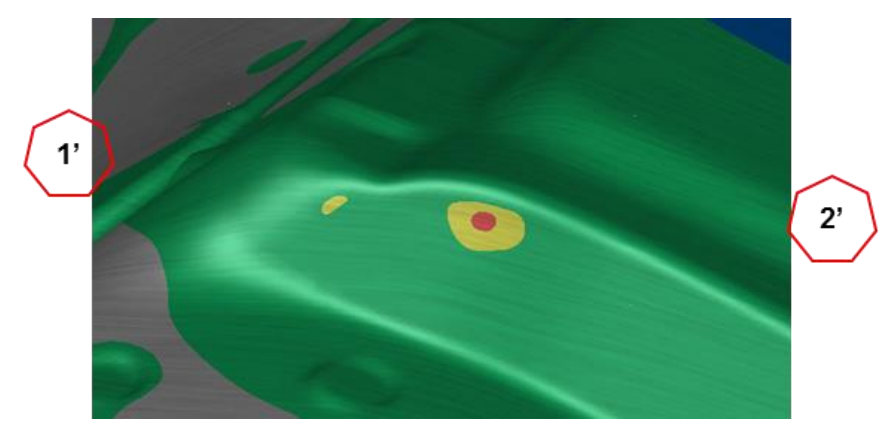

Figure 16. Zoom des Zones à risque de casse

\subsubsection{Déformation plastique}

La variable de résultat (Plastic Strain) peut être utilisée comme indication de déformation plastique permanente, en particulier pour les pièces de peau, le fléchissement du flan et la fermeture du serre-flan ne doivent pas produire de plis. Dans ce cas de simulation, elle devrait être inférieure à $0,2 \%$.

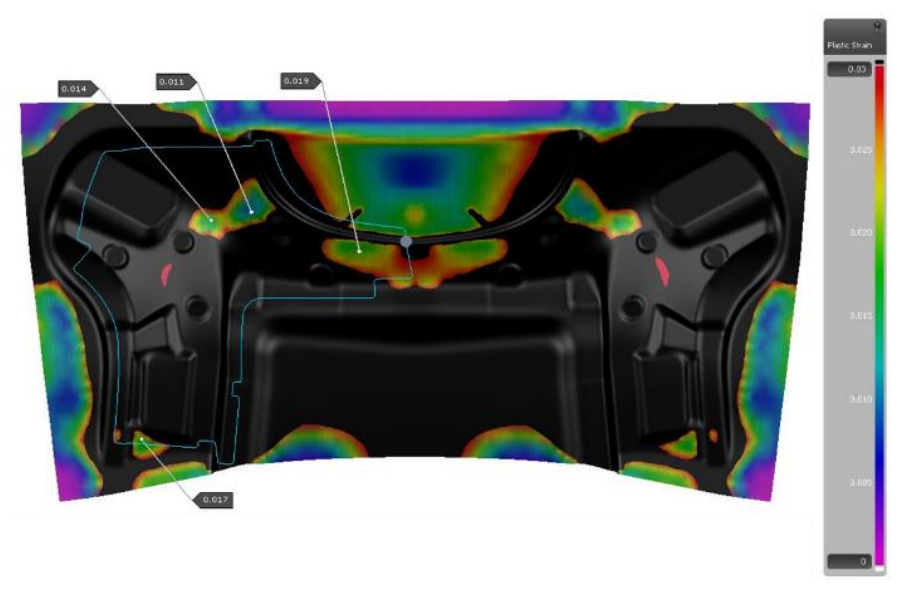

Figure 17. Déformation plastique

\subsubsection{Ondulation/Plis}

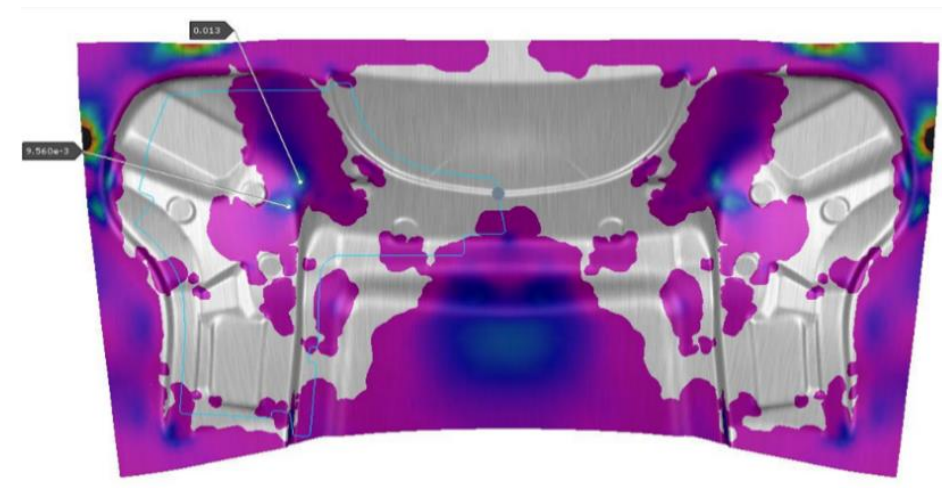

Figure 18. Ondulation/Plis au nominal 
Les défauts d'aspects tels que les ondulations et plis ne sont pas autorisées sur une pièce. La figure 18 montre qu'elle y a des zones avec épaississement/ondulations, pourtant ce sont des zones de Serreflan ou bien d'habillage nos aspects qui appartient à la surface annexe autre que la DFN qui représente le produit fini.

\subsubsection{Amincissements}

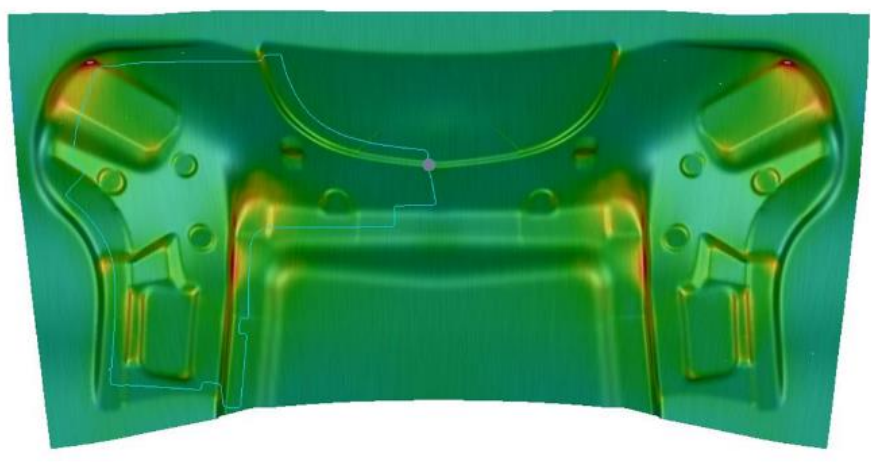

Figure 19. Amincissements

La variable de résultat Thinning représente la distribution d'épaisseur de la pièce. Dans l'industrie automobile, il existe des exigences concernant la réduction d'épaisseur maximale admissible, afin de garantir des marges de sécurité en cas de choc.

\section{Conclusion}

La simulation numérique de la tôle est devenue incontournable par la plupart des constructeurs automobiles et des principaux fabricants d'outils. L'un des principaux avantages de la simulation de formage de tôles est qu'elle permet de tester des formes de pièces très compliquées ce qui conduit à une réduction significative à la fois du coût et du temps par rapport à la mise au point par essai-erreur. A condition que les paramètres engagés démontrent une bonne correspondance entre la simulation et le procédé de production réel. La prévision de la striction localisée et la rupture dans les opérations de formage de tôles est cruciale pour obtenir la robustesse de la gamme d'emboutissage avec une qualité géométrique du produit embouti élevé par rapport a la définition numérique. Dans cet article, un modèle de prédiction par le biais d'Autoform est étudié afin de prédire la striction localisée dans une pièce automobile. Le modèle est purement basé sur des données déterminées lors d'essais de traction uni-axiaux. Les données concernant le risque de fracture sont obtenues en créant une courbe limite de formation (FLC).

\section{Bibliographie}

[AND 04] A. ANDERSSON, « Comparison of sheet-metal-forming simulation and try-out tools in the design of a forming tool », Journal of Engineering Design, vol. 15, no. 6. pp. 551-561, 2004.

[RAD 16] B. RADI AND A. El HAMI, Material Forming Processes: Simulation, Drawing, Hydroforming and Additive Manufacturing, ISTE Editions, Londres, 2017.

[HAM 16] F. HAMZA, O. BousSAID, AND K. TADJINE, «Finite element analysis of the wheelbarrows tear during the metal forming », Proceedings of The IRES 25th International Conference, Istanbul, Turkey, 24th January 2016.

[DRO 18] K. DROTLEFF AND M. LIEWALD, « Application of an advanced necking criterion for nonlinear strain paths to a complex sheet metal forming component », IOP Conference Series: Materials Science and Engineering, vol. 418, no. $1,2018$. 
[ABB 85] F. AbBassi, O. PANTAle, A. Zghal, S. Mistou, AND R. RAKotomalala, « L'étude de comportement élastoplastique anisotrope de tôle d'emboutissage par l'utilisation de corrélation d'image (DIC) », 1885.

[BEN 17] K. BENOTHMEN, P. Y. MANACH, AND K. ELLEUCH, «Influence du comportement matériau (anisotropie et écrouissage) lors de la mise en forme par emboutissage inverse Abstract», Lille, 28 Août au 1er Septembre 2017.

[RAD 07] B. RADI AND A. EL HAMI, « Reliability analysis of the metal forming process », International Journal of Mathematical and Computer Modelling, vol. 45, no. 3-4, pp. 431-439, 2007.

[OZT 04] F. OZTURK AND D. LEE, « Analysis of forming limits using ductile fracture criteria » Journal of Materials Processing Technology, vol. 147, no. 3, pp. 397-404, Apr. 2004.

[MAT 00] K. MATTIASSON, « On finite element simulation of sheet metal forming processes in industry », in European Congress on Computational Methods in Applied Sciences and Engineering, ECCOMAS 2000, pp. 11-14, Barcelona, 1114 September 2000.

[MAK 98] A. MAKINOUCHI, C. TEODOSIU, AND T. NAKAGAWA, « Advance in FEM simulation and its related technologies in sheet metal forming», CIRP Annals - Manufacturing Technology, vol. 47, no. 2, pp. 641-649, 1998.

[KUB 95] W. KUBLI AND J. REISSNER, « Optimization of sheet-metal forming processes using the special-purpose program AUTOFORM », Journal of Materials Processing Technology, vol. 50, no. 1-4, pp. 292-305, 1995.

[LOU 12] Y. LOU, H. HUH, S. LIM, AND K. PACK, « New ductile fracture criterion for prediction of fracture forming limit diagrams of sheet metals », International Journal of Solids and Structures, vol. 49, no. 25, pp. 3605-3615, 2012.

[FED 17] M FEDorko, M URBÁNeK, M RUND, «Prediction of thinning of the sheet metal in the program AutoForm and its experimental », Conf. Series: Materials Science and Engineering 179, Czech Republic, 2017. 